\title{
Diagnostic performance of a CT-based scoring system for diagnosis of anastomotic leakage after esophagectomy: comparison with subjective CT assessment
}

\author{
Lucas Goense $^{1,2}$ (1) • Pauline M. C. Stassen ${ }^{1} \cdot$ Frank J. Wessels $^{3}$. \\ Peter S. N. van Rossum ${ }^{1,2}$ • Jelle P. Ruurda ${ }^{1}$ • Maarten S. van Leeuwen ${ }^{3}$. \\ Richard van Hillegersberg ${ }^{1}$
}

Received: 5 October 2016 / Revised: 20 January 2017 / Accepted: 13 March 2017 / Published online: 29 March 2017

(C) The Author(s) 2017. This article is published with open access at Springerlink.com

\begin{abstract}
Objective To develop a CT-based prediction score for anastomotic leakage after esophagectomy and compare it to subjective CT interpretation.

Methods Consecutive patients who underwent a CT scan for a clinical suspicion of anastomotic leakage after esophagectomy with cervical anastomosis between 2003 and 2014 were analyzed. The CT scans were systematically re-evaluated by two radiologists for the presence of specific CT findings and presence of an anastomotic leak. Also, the original CT interpretations were acquired. These results were compared to patients with and without a clinical confirmed leak.

Results Out of 122 patients that underwent CT for a clinical suspicion of anastomotic leakage; 54 had a confirmed leak. In multivariable analysis, anastomotic leakage was associated with mediastinal fluid $(\mathrm{OR}=3.4)$, esophagogastric wall discontinuity $(\mathrm{OR}=4.9)$, mediastinal air $(\mathrm{OR}=6.6)$, and a fistula
\end{abstract}

Maarten S. van Leeuwen and Richard van Hillegersberg contributed equally to this work.

\section{Lucas Goense}

L.Goense-2@umcutrecht.nl

Richard van Hillegersberg

R.vanHillegersberg@umcutrecht.nl

1 Department of Surgery, University Medical Center Utrecht, G.04.228, Heidelberglaan 100, 3584CX Utrecht, The Netherlands

2 Department of Radiation Oncology, University Medical Center Utrecht, Utrecht, The Netherlands

3 Department of Radiology, University Medical Center Utrecht, Utrecht, The Netherlands
$(\mathrm{OR}=7.2)$. Based on these criteria, a prediction score was developed resulting in an area-under-the-curve (AUC) of 0.86 , sensitivity of $80 \%$, and specificity of $84 \%$. The original interpretation and the systematic subjective CT assessment by two radiologists resulted in AUCs of 0.68 and 0.75 with sensitivities of $52 \%$ and $69 \%$, and specificities of $84 \%$ and $82 \%$, respectively.

Conclusion This CT-based score may provide improved diagnostic performance for diagnosis of anastomotic leakage after esophagectomy.

Key Points

- A CT-based score provides improved diagnostic performance for diagnosis of anastomotic leakage.

- Leakage associations include mediastinal fluid, mediastinal air, wall discontinuity, and fistula.

- A scoring system yields superior diagnostic accuracy compared to subjective CT assessment.

- Radiologists may suggest presence of anastomotic leakage based on a prediction score.

Keywords Esophagectomy · Anastomotic leakage · Prediction score $\cdot$ Computed tomography $\cdot$ Esophageal cancer

\begin{tabular}{ll}
\multicolumn{2}{l}{ Abbreviations } \\
ALP-score & anastomotic leakage prediction score \\
AUC & area-under-the-curve \\
CI & confidence interval \\
CT & computed tomography \\
CRP & C-reactive protein \\
NRI & net reclassification index \\
OR & odds ratio \\
WBC & White blood cell count
\end{tabular}




\section{Introduction}

Esophageal cancer is the sixth leading cause of cancer-related mortality worldwide and the incidence rate is rapidly increasing [1]. Surgical resection of the esophagus with en-bloc lymphadenectomy combined with neoadjuvant chemoradiation or perioperative chemotherapy is the cornerstone of treatment for patients with locally advanced non-metastatic esophageal cancer [2-4]. Despite advances in surgical treatment and improvement in perioperative care, anastomotic leakage remains a frequently encountered complication after esophagectomy with reported frequency rates of up to $30 \%[2,5]$. Early detection of anastomotic leakage is crucial, since delayed treatment is associated with significant morbidity, prolonged hospital stay, and mortality [6-9].

Several diagnostic modalities are available in case anastomotic leakage is clinically suspected, such as contrast swallow examination, endoscopy, or computed tomography (CT). Contrast swallow examinations are widely performed in order to assess anastomotic integrity. Although contrast swallow examinations are very specific, multiple studies have shown that they are of poor sensitivity, failing to identify significant anastomotic leaks [10-13]. Meanwhile, endoscopy after esophagectomy has proven to be a more accurate method to diagnose anastomotic leakage and provide information on the condition of the gastric tube $[14,15]$. However, most physicians are reluctant to utilize endoscopic examination early after esophagectomy as this invasive procedure may damage the anastomosis.

CT scanning is commonly performed for diagnosis of postoperative complications, since it is non-invasive and safe to use in critically ill patients. Previously, several studies have assessed the usefulness of CT scanning for the detection of anastomotic leakage after esophagectomy [13, 16-18]. However, most of these studies assessed the diagnostic value of CT during postoperative routine screening and included only a small number of patients $[13,16,17]$. Also, a wide range of diagnostic accuracies has been reported, suggesting that the association of different radiological findings after esophagectomy with anastomotic leakage is unclear [16-18].

Previous studies have shown that assessment of specific CT findings was useful for the prediction of anastomotic leakage after gastric and colorectal surgery $[19,20]$.

In summary, objective criteria to detect anastomotic leakage on CT have not been clearly defined. Therefore, the purpose of this study was to determine reliable CT findings that can be used to diagnose anastomotic leakage and develop a CT-based risk prediction score for confirming or ruling out anastomotic leakage in a large cohort of patients with a clinical suspicion of leakage after esophagectomy. Also, the diagnostic performance of this CT-based risk prediction score was compared to that of a systematic subjective evaluation by two expert radiologists and that of the original $\mathrm{CT}$ interpretation.

\section{Materials and methods}

\section{Study population}

This retrospective cohort study was approved by an institutional review board and the requirement to obtain informed consent was waived. The study was designed and conducted according to Standards for Reporting of Diagnostic Accuracy [21]. From a prospectively acquired database, all consecutive patients with esophageal or gastro-esophageal junction cancer who underwent an elective esophagectomy, between 2003 and 2014, at our tertiary referral center were identified. Within this database patients who were evaluated with a CT scan for a clinically suspected anastomotic leak after elective esophageal surgery were included. Surgical treatment consisted of a transthoracic or transhiatal esophagectomy with en-bloc lymphadenectomy and gastric tube reconstruction [22]. A cervical esophagogastric anastomosis was performed end-toside with hand-sewn continuous sutures (3-0 PDS) in monolayer. After surgery two chest tubes were routinely placed, and removed during the following days in case of limited drainage $(<200 \mathrm{ml} / 24 \mathrm{~h})$, and absence of air leak.

\section{Data collection}

Clinical patient characteristics were extracted from the prospectively maintained database (Table 1 ). In addition, heart rate, temperature, white blood cell count (WBC) and Creactive protein (CRP) were extracted from the patients' charts on the day anastomotic leakage was clinically suspected (day of CT scan). Anastomotic leakage was confirmed by either postoperative demonstration of saliva through the cervical wound, or visualization of anastomotic dehiscence or fistula during endoscopy or surgical reintervention. The follow-up time was truncated to 30 days for all patients. All postoperative complications, including anastomotic leakage, were prospectively registered.

\section{Image acquisition}

Thoraco-abdominal CT images were acquired using commercially available 16- or 64-section CT scanners (Philips Medical Systems, Best, The Netherlands). Images were typically acquired with $64 \times 0.625$ millimeter section collimation, a tube rotation time of 500 milliseconds, a tube potential of 100 or $120 \mathrm{kV}$, an effective tube current of $120 \mathrm{mAs}$, and a pitch of 0.9 or 1.1. An iodinated $90-\mathrm{mL}$ contrast material bolus was administered intravenously at $4 \mathrm{~mL} / \mathrm{sec}$ in all patients. Oral contrast intake was not routinely used in our center as this was shown to have limited sensitivity for the detection of anastomotic leakage [13]. 
Table 1 Clinical and treatment-related characteristics in relation to anastomotic leakage

\begin{tabular}{lccc}
\hline Characteristic & $\begin{array}{c}\text { Anastomotic } \\
\text { leakage }(n=54)\end{array}$ & $\begin{array}{c}\text { No anastomotic } \\
\text { leakage }(n=68)\end{array}$ & $p$ value \\
\hline Male gender & $41(75.9)$ & $56(82.4)$ & 0.382 \\
Age (years)* & $65.2 \pm 9.0$ & $65.8 \pm 8.9$ & 0.708 \\
BMI $\left(\mathrm{kg} / \mathrm{m}^{2}\right)^{*}$ & $25.7 \pm 4.4$ & $26.8 \pm 4.3$ & 0.158 \\
ASA score & & & 0.841 \\
I & $12(22.2)$ & $12(17.6)$ & \\
II & $28(51.9)$ & $40(58.8)$ & \\
III & $14(25.9)$ & $15(22.1)$ & \\
IV & $0(0.0)$ & $1(1.5)$ & \\
COPD & $13(24.1)$ & $10(14.7)$ & 0.189 \\
Cardiac co-morbidity & $15(27.8)$ & $23(33.8)$ & 0.474 \\
Diabetes mellitus & $9(16.7)$ & $15(22.1)$ & 0.457 \\
Current smoker & $17(31.5)$ & $21(30.9)$ & 0.898 \\
Neoadjuvant therapy & & & 0.973 \\
$\quad$ None & $16(30.9)$ & $21(29.6)$ & \\
Chemotherapy & $12(22.6)$ & $14(21.2)$ & \\
$\quad$ Chemoradiotherapy & $26(49.1)$ & $33(50.0)$ & \\
Heart rate*广 & $103 \pm 22$ & $96 \pm 19$ & 0.085 \\
Temperature* & $37.7 \pm 0.8$ & $37.7 \pm 0.8$ & 0.950 \\
Leukocytes*\& & $16.2 \pm 7.1$ & $15.6 \pm 7.3$ & 0.385 \\
C-reactive protein*\| & $224 \pm 104$ & $194 \pm 96$ & 0.110 \\
\hline
\end{tabular}

Note. Data are numbers of patients with percentages in parentheses

* Data are mean \pm standard deviation

${ }^{\dagger}$ Heart rate in beat per minute

Temperature in Celsius $\left({ }^{\circ} \mathrm{C}\right)$

${ }^{\S}$ Number of leukoctyes $\times 10^{9} / \mathrm{L}$

${ }^{\|} \mathrm{CRP}$ in $\mathrm{mg} / \mathrm{L}$

\section{Variable selection}

CT findings related to anastomotic leakage and esophageal surgery were selected for image analysis by two radiologists and two gastrointestinal surgeons during a consensus meeting. On the basis of their clinical experience, the most frequently encountered CT findings following esophageal surgery and variables described previously in the literature were included for analysis. The selected CT findings included mediastinal fluid collection, mediastinal air, mediastinal abscess (i.e., central zone of necrotic inflammatory material encapsulated by a discernible wall), and mediastinal induration (whenever the mediastinal fat showed non-contiguous, patchy inhomogeneity with water or low-Hounsfield units soft tissue density $[<20$ Hounsfield units]). When a mediastinal collection was present, the frequency, size of the largest collection, and the anatomical region (i.e., above the manubrium, between the manubrium and carina, between the carina and diaphragm, and below the diaphragm) were recorded. Other CT findings included a visible discontinuity of the esophagogastric wall, a fistula (scored if a fluid- or air-filled tract was visible between the esophagogastric anastomosis and another anatomic cavity [skin, trachea, pleural cavity or mediastinum]), pleural effusion, empyema (atypical pleural effusion, loculation in the pleural space, and thickening of the pleural membranes) and presence of a lung consolidation (i.e., atelectasis, pneumonia or non-specific).

\section{Image evaluation}

All CT scans were retrospectively reviewed together by two radiologists in consensus (MSL and FJW with more than 25 and 6 years experience in gastrointestinal imaging, respectively). Images were reviewed on a picture archiving and communication system (Sectra AB, version 17.3, Linköping, Sweden). The reviewers knew that all patients had been subjected to an esophagectomy, but were blinded for the patients' detailed clinical information. The presence or absence of the various selected CT variables were systematically assessed and recorded. After the systematic assessment of the CT findings, the reviewers also indicated if they suspected the patient to have an anastomotic leak (i.e., no anastomotic leak, a probable leak, or a definite anastomotic leak), further referred to as the systematic subjective assessment.

Also, all the original CT interpretations rendered as part of the clinical care were reviewed. Each CT report was originally interpreted by a board certified radiologist. The original interpretations were classified as 'no leak, 'probable leak', or 'definite leak'.

\section{Statistical analysis and development of a practical scoring system}

The association of clinical patient characteristics with anastomotic leakage was studied univariably. Categorical parameters were compared using the chi-square test or Fisher's exact test in case of small cell counts. Student's T-test and the MannWhitney- $U$ test were used to compare groups with and without anastomotic leakage for parametric and non-parametric continuous parameters, respectively. In order to analyze whether the different CT findings were associated with anastomotic leakage, univariable logistic regression models were used providing odds ratios (ORs) with $95 \%$ confidence intervals (CIs). Subgroup analyses were performed to assess whether location in the mediastinum and days after surgery influenced the associations of the different CT findings with anastomotic leakage.

Subsequently, parameters with a $p$-value below 0.05 in univariable logistic regression analysis were entered into a multivariable logistic regression model with backward stepwise selection to evaluate whether these factors were independently associated with the occurrence of anastomotic leakage. A practical scoring system was developed using the beta-regression coefficients of the retained predictive factors. 
To compare diagnostic performances of the different CT assessments, receiver operating characteristics (ROC) curve analysis was performed and the area-under-the-curves (AUC) were computed. Ideal cut-off values were calculated by giving equal weight to sensitivity and specificity. In addition, the potential superiority of the prediction score in comparison with the systematic subjective and original assessment was evaluated using the net reclassification index (NRI). The NRI reflects the reclassification ability of the model and is the sum of improvement in correctly predicting patients with and without leakage [23]. Statistical analysis was performed using SPSS version 23.0 (IBM Corp., Armonk, NY). A $p$-value of $<0.05$ was considered statistically significant.

\section{Results}

\section{Demographics}

In the study period, a total of 405 patients underwent esophagectomy with gastric tube reconstruction. Of these patients, 283 were excluded because there was no clinically suspected leak ( $n=238)$, no CT scan was performed in case of a suspected anastomotic leak $(n=43)$ or the CT scan was of insufficient quality $(n=2)$. Consequently, 122 patients were deemed eligible for inclusion in our study, of whom 54 (44.3\%) had a confirmed anastomotic leak (Fig. 1).

Clinical and treatment-related patient characteristics and their univariable association with anastomotic leakage are summarized in Table 1. None of the studied patient and treatment-related factors were significantly associated with the occurrence of anastomotic leakage. The median time interval between esophagectomy and CT acquisition was 6 days (range: 1-32). Anastomotic leakage occurred after a median time of 8 days (range: 1-22) following esophagectomy. Anastomotic leakage was confirmed by, endoscopy $(n=10)$,

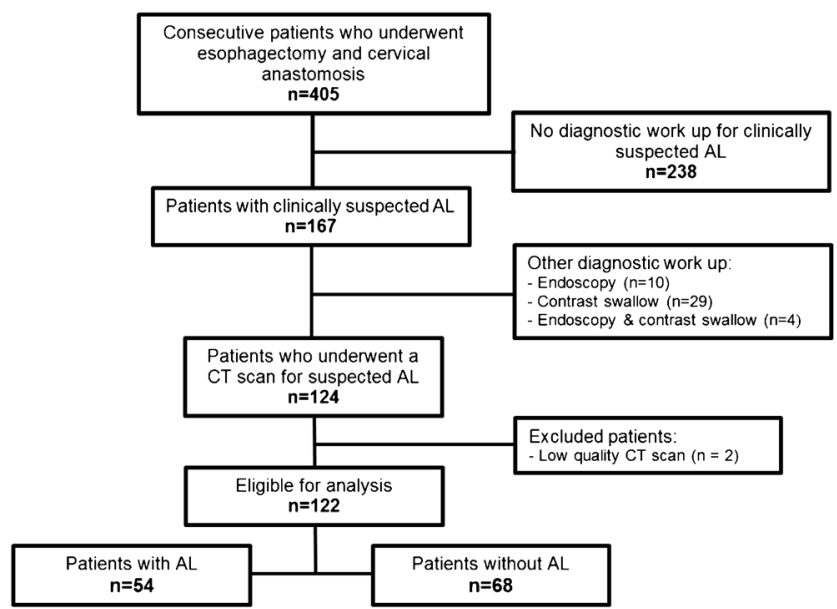

Fig. 1 Flowchart demonstrates the selection process of patients with a suspicion of anastomotic leakage (AL) surgical re-intervention $(n=31)$ or demonstration of saliva during opening of the cervical wound $(n=13)$. Treatment of anastomotic leakage consisted of ceasing oral intake in combination with opening of the cervical wound $(n=20)$, placing a stent $(n=3)$ or surgical re-intervention $(n=31)$.

\section{Predictors of anastomotic leakage}

The results of univariable logistic regression analyses for each specific CT finding in relation to anastomotic leakage are presented in Table 2. In univariable analyses studying specific CT findings, the presence of a mediastinal fluid collection (OR 3.1, 95\% CI: 1.4-7.1, $p=0.006$ ) and mediastinal air (OR 11.1, 95\% CI: 3.6-34.2, $p<0.001$ ) were significantly associated with anastomotic leakage.

In subgroup analyses the associations of mediastinal fluid with anastomotic leakage was independent of its size, anatomic location within the mediastinum, and the number of days they occurred after surgery. In subgroup analyses of patients with presence of mediastinal air on their postoperative CT scan $(70 \%, 86 / 122)$, the number of days after surgery that air was observed was significantly associated with anastomotic leakage (OR for each additional postoperative day: 1.162, 95\% CI: 1.022-1.327, $p=.022)$. To this regard, the prevalence of a confirmed leak in patients with observed free air before or after the seventh postoperative day was $50 \%$ and $73 \%$, respectively. Of the patients with free air in the mediastinum, air was observed above the manubrium in 22 patients $(12 / 22$, $55 \%$ leakage), between the manubrium and carina in 4 patients (3/4, 75\% leakage), between carina and diaphragm in eight patients $(4 / 8,50 \%$ leakage), and in a combination of these anatomic locations in 52 patients (31/52, 60\% leakage). The association of mediastinal air with anastomotic leakage was independent of its location $(p=0.838)$. Also the size of mediastinal air on CT was not associated with anastomotic leakage.

In addition, the presence of wall discontinuity (OR 12.6, 95\% CI: 4.39-36.20, $p<0.001$ ), fistula (OR 12.7, 95\% CI: $2.8-58.5, p<0.001)$ and empyema (OR 17.1, 95\% CI: $2.1-$ $137.6, p=0.007$ ) were significantly associated with anastomotic leakage. No significant difference in incidence of other CT findings among patients with or without anastomotic leakage was found.

In multivariable logistic regression analysis, a mediastinal fluid collection (OR 3.4, 95\% CI: 1.3-9.4, $p=0.016$ ), mediastinal air (OR 6.6, 95\% CI: 1.9-23.2, $p=0.003$ ), wall discontinuity (OR 4.9, 95\% CI: 1.5-15.9, $p=0.008$ ), and presence of a fistula (OR 7.2, 95\% CI: $1.2-43.8, p=0.032$ ) remained independently and significantly associated with anastomotic leakage (Table 3, Fig. 3). The association between empyema and anastomotic leakage was no longer significant after multivariable adjustment $(p=0.093)$. 
Table 2 Univariable logistic regression analysis of specific postoperative CT findings in relation to anastomotic leakage after esophagectomy

\begin{tabular}{lcccc}
\hline Characteristic & $\begin{array}{l}\text { Anastomotic } \\
\text { leakage }(n=54)\end{array}$ & $\begin{array}{l}\text { No anastomotic } \\
\text { leakage }(n=68)\end{array}$ & OR (95\% CI) & $p$ value \\
\hline Mediastinal: & & & & \\
$\quad$ Induration & $7(13.0)$ & $6(8.8)$ & $1.5(0.49-4.88)$ & 0.464 \\
$\quad$ Fluid collection & $23(42.6)$ & $13(19.1)$ & $3.1(1.40-7.06)$ & $0.006^{*}$ \\
$\quad$ Abscess & $7(13.0)$ & $4(5.9)$ & $2.4(0.66-8.61)$ & 0.185 \\
$\quad$ Air & $50(92.6)$ & $36(52.9)$ & $11.1(3.61-34.20)$ & $<0.001^{*}$ \\
Wall discontinuity & $27(50.0)$ & $5(7.4)$ & $12.6(4.39-36.20)$ & $<0.001^{*}$ \\
Fistula & $15(27.8)$ & $2(2.9)$ & $12.7(2.76-58.47)$ & $<0.001^{*}$ \\
Pleural effusion & $46(85.2)$ & $58(85.3)$ & $1.0(0.36-2.71)$ & 0.987 \\
Empyema & $11(20.4)$ & $1(1.5)$ & $17.1(2.1-137.6)$ & $0.007^{*}$ \\
Atelectasis & $50(92.6)$ & $59(86.8)$ & $1.9(0.55-6.57)$ & 0.306 \\
Pulmonary infiltrate & $11(20.4)$ & $19(27.9)$ & $0.7(0.28-1.54)$ & 0.336 \\
\hline
\end{tabular}

Note - Data presented as counts with percentages in the parentheses

*Significant difference between patients with versus without anastomotic leakage $(p<0.05)$

OR: odds ratio. CI: confidence interval

\section{Systematic subjective CT assessment}

During systematic subjective CT scan assessment by the radiologists, a leak was suggested in 49 patients of which 37 (75.5\%) had a confirmed leak, whereas absence of a leak was scored in 53 patients of which ten $(18.9 \%)$ had a confirmed leak. Of the remaining patients with a probable leak $(n=20), 7(35 \%)$ had a confirmed leak. The radiologists evaluation, referred to as 'systematic subjective assessment', yielded an AUC of 0.75 (95\% CI: 0.66-0.84) in ROC analysis (Fig. 2, Table 4). Sensitivity and specificity of the systematic subjective assessment by the radiologists (no + probable leakage versus presence of leakage) were $68.5 \%$ ( 37 of $54 ; 95 \%$ CI: $54.3-80.1$ ) and $82.4 \%$ (56 of 68; 95\% CI: 70.8-90.1), respectively (Table 4).

\section{Original clinical CT interpretation}

The original clinical CT interpretation yielded an AUC of 0.68 (95\% CI: 0.59-0.78) in ROC analysis (Fig. 2, Table 4). Sensitivity and specificity of the original assessment by the radiologists (absence of leakage versus probable + presence of leakage) were $51.9 \%$ (28 of 54; $95 \%$ CI: 38.0-65.5) and $83.8 \%$ (57 of $68 ; 95 \%$ CI: 72.5-91.3), respectively (Table 4).

\section{Risk scoring system}

An anastomotic leakage prediction score (ALP score) was constructed based on the four $\mathrm{CT}$ findings that remained significantly associated with anastomotic leakage in multivariable analysis. Based on the absolute beta-regression coefficient, presence of each variable was converted into a corresponding amount of points rounded to its nearest integer. Next scaling was performed with respect to the discriminatory power of the scores as determined by ROC analysis. To this regard it proved feasible to assign one point for the presence of each predictive factor - in order to keep the score simple - without compromising its discriminative ability (Table 3). Therefore, the cumulative amount of points of the ALP score ranges from 0 to 4 . The diagnostic performance of the possible scores for identifying an anastomotic leak are presented in Table 5.
Table 3 Multivariable logistic regression analysis of CT findings significantly associated with anastomotic leakage in univariable analysis

\begin{tabular}{lllll}
\hline Characteristic & $\begin{array}{l}\beta \text { regression } \\
\text { coefficient }\end{array}$ & OR $(95 \%$ CI $)$ & $p$ value & Points $^{\dagger}$ \\
\hline Fluid collection & 1.233 & $3.43(1.26-9.34)$ & $0.016^{*}$ & 1 \\
Air cavity & 1.882 & $6.57(1.86-23.21)$ & $0.003^{*}$ & 1 \\
Wall discontinuity & 1.591 & $4.91(1.52-15.88)$ & $0.008^{*}$ & 1 \\
Fistula & 1.973 & $7.19(1.18-43.84)$ & $0.032^{*}$ & 1 \\
Empyema & 1.987 & $7.29(0.72-74.30)$ & 0.093 & $\mathrm{NA}$ \\
\hline
\end{tabular}

* Significant difference between patients with versus without anastomotic leakage $(p<0.05)$

OR: odds ratio, CI: confidence interval

${ }^{\dagger}$ Assignment of points to CT findings was based the corresponding $\beta$ regression coefficient. Scaling was performed with respect to the discriminatory power of the scores as determined by ROC analysis 


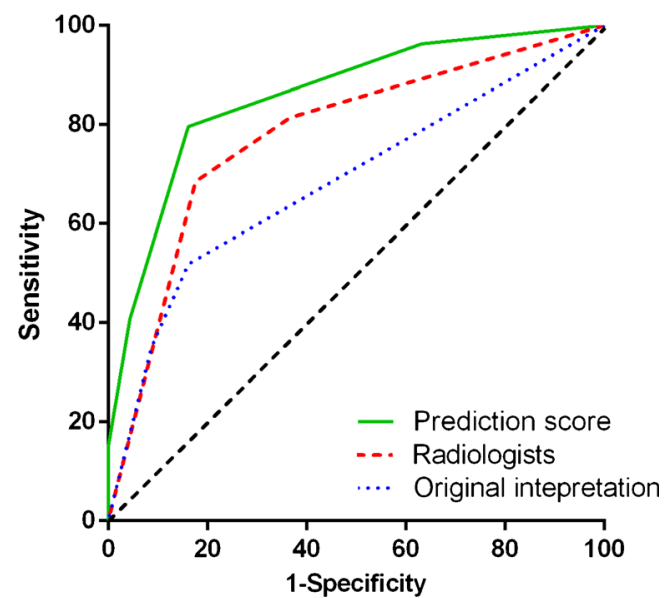

Fig. 2 ROC curve analysis of the 'anastomotic leakage prediction score' (green line), the systematic subjective CT assessment by expert radiologists (red dotted line) and the original interpretation (blue dotted line) indicating their ability to discriminate between patients with and without anastomotic leakage

Using ROC analysis a total of two points was statistically determined as optimal cut-off, in which patients with scores $\geq 2$ points were considered at high risk of anastomotic leakage. The cut-off value of $\geq 2$ points yielded a sensitivity of $80 \%$ ( 43 of $54 ; 95 \%$ CI: $66.1-88.9$ ) and specificity of $84 \%$ (57 of 68 ; 95\% CI: 72.5-91.3). The final ALP score model had an AUC of 0.86 (95\% CI: 0.79-0.93) (Fig. 2, Table 4).

The ALP scoring system improved the AUC ( 0.86 versus 0.75 and 0.68$)$ with an NRI of $12.5 \%(p=0.008)$ and $27.7 \%$ $(p<0.001)$ for the detection of anastomotic leakage compared to the systematic subjective $\mathrm{CT}$ assessment and original CT interpretation, respectively (Table 4). These findings indicate that with the ALP score $11.1 \%$ and $27.7 \%$ of the patients with definite anastomotic leakage, and $1.4 \%$ and $0 \%$ of patients without leakage were better classified compared to the systematic subjective $\mathrm{CT}$ assessment and original CT interpretation, respectively (Fig. 3).

\section{Discussion}

In this retrospective cohort study, CT findings of patients with a clinically suspected anastomotic leak after esophagectomy were systematically analyzed and predictors of anastomotic leakage were identified. Presence of mediastinal fluid, mediastinal air, esophagogastric wall discontinuity and fistula were independently associated with anastomotic leakage, irrespective of clinical and treatment-related patient characteristics. Based on these CT findings, a prediction score for anastomotic leakage was developed. The 4-point ALP score demonstrated good diagnostic performance. This study demonstrates superior diagnostic accuracy of a CT-based scoring system in comparison with the systematic subjective assessment (NRI: $12.5 \%$ ), and original CT interpretation (NRI: $27.7 \%$ ) of leakage on a post-esophagectomy CT scan. The easy to use point-based ALP score may provide radiologists and surgeons a tool to objectively assess the risk of anastomotic leakage after esophageal surgery in patients with a suspicion of such complication.

CT scanning is increasingly being performed for the detection of anastomotic leakage after esophagectomy, since it is non-invasive, safe in critically ill patients, and aids in the detection of other associated findings (e.g., pulmonary complications) [16, 17]. However, the interpretation of a CT scan after prior esophageal resection remains difficult due to the anatomic changes and residual air and fluid shortly after surgery. Previous studies have assessed the diagnostic value of CT scanning for the detection of anastomotic leakage after esophagectomy [13, 16-18]. In most of these studies, one or two reviewers determine their own definition as to what an anastomotic leak on a CT scan consists of, without assessing specific CT findings [16, 17]. In the literature this results in a large difference in reported diagnostic values $[16,17]$. This observation is confirmed by the current study in which a difference of $17 \%$ in sensitivity was found between the original CT interpretation and the systematic subjective CT assessment. These findings are suggestive for a lack of consensus on radiographic findings associated with anastomotic leakage in patients after esophagectomy.

Until now, two studies have made a similar attempt to identify specific CT findings for the detection of anastomotic leakage after esophagectomy [13, 18]. One study that included 97 patients assessed several specific CT

Table 4 Receiver operating characteristics analysis and net reclassification index (NRI) estimates for anastomotic leakage according to the original interpretation, subjective $\mathrm{CT}$ assessment and the anastomotic leakage prediction model

\begin{tabular}{|c|c|c|c|c|c|c|c|}
\hline Model & $\operatorname{AUC}(95 \% \mathrm{CI})$ & Ideal cut-off & $\mathrm{SE}(\%)$ & $\mathrm{SP}(\%)$ & $\operatorname{PPV}(\%)$ & $\operatorname{NPV}(\%)$ & NRI $(\%)$ \\
\hline Original CT interpretation & $0.68(0.59-0.78)$ & No AL vs. probable or definite AL & 51.9 & 83.8 & 71.8 & 68.7 & reference \\
\hline Systematic assessment & $0.75(0.66-0.84)$ & No or probable AL vs. definite AL & 68.5 & 82.4 & 75.5 & 76.7 & 15.2 \\
\hline ALP-score model & $0.86(0.79-0.92)$ & Score $\geq 2$ vs. score $<2$ & 79.6 & 83.8 & 81.1 & 83.8 & 27.7 \\
\hline
\end{tabular}

ALP-score: anastomotic leakage prediction score. AL: anastomotic leakage. AUC: area under the curve. SE: sensitivity. SP: specificity. PPV: positive predictive value. NPV: negative predictive value. NRI: Percentage of net reclassification index 
Table 5 Risk scores and their coordinates on the ROC curve

\begin{tabular}{lclcc}
\hline Risk score & $\mathrm{n}$ & Observed leakage risk & Sensitivity* (\%) & Specificity* (\%) \\
\hline Anastomotic leakage prediction score & & & \\
ALP score 0 & 27 & $7.4 \%(2 / 27)$ & 100 & 0 \\
ALP score 1 & 41 & $22.0 \%(9 / 41)$ & 96.3 & 36.8 \\
ALP score 2 & 29 & $72.4 \%(21 / 29)$ & 79.6 & 83.8 \\
ALP score 3 & 17 & $82.4 \%(14 / 17)$ & 40.7 & 95.6 \\
ALP score 4 & 8 & $100 \%(8 / 8)$ & 14.8 & 100 \\
Systematic subjective assessment & & & 0 \\
No leakage & 53 & $18.9 \%(10 / 53)$ & 100 & 63.2 \\
Probable leakage & 20 & $35.0 \%(7 / 20)$ & 81.5 & 82.4 \\
Presence of leakage & 49 & $75.5 \%(37 / 49)$ & 68.5 & 0 \\
Original CT interpretation & & & & \\
No leakage & 83 & $31.3 \%(26 / 83)$ & 100 & 93.8 \\
Probable leakage & 14 & $64.3 \%(9 / 14)$ & 35.2 & 91.2 \\
Presence of leakage & 25 & $76.0 \%(19 / 25)$ &
\end{tabular}

ROC: receiver operating characteristics

* Sensitivity and specificity defined by their coordinates on the ROC curve findings during postoperative routine screening. In that study presence of mediastinal air and contrast leakage on postoperative day 3 and 7 were associated with anastomotic leakage [13]. Another study that included 54 patients found mediastinal air and mediastinal fluid to be associated with anastomotic leakage [18]. These observations partially correspond with the results of our study that found an association of anastomotic leakage with mediastinal air and fluid collections. However, in these two previously mentioned studies only few events of anastomotic leakage occurred ( $n=11$ and $n=6$, respectively), which results in an uncertainty of estimates [13, 18].
Interestingly, these studies show that using solitary CT findings as diagnostic marker, without combining them in a model, results in either low sensitivity or low specificity. Contrast leakage after esophagectomy, for example, is known to be a specific finding but the absence of extravasation of contrast is associated with high falsenegative rates and consequently a low sensitivity [13]. On the contrary, presence of mediastinal air near the gastric tube is very sensitive, but since this is a common finding after esophageal surgery it is not very specific $[13,18]$. Our data suggest that combining specific CT findings in a risk score could be used to overcome these
Fig. 3 Examples of CT findings associated with the presence of anastomotic leakage after esophagectomy. A: Image shows a fistula between the gastric tube and right pleural cavity (arrow). B: Image shows a fluid collection (arrow) in the mediastinum. C: Image shows a visible discontinuity of the esophagogastric wall (arrow). D: Image shows a mediastinal air cavity (arrow) after esophagectomy
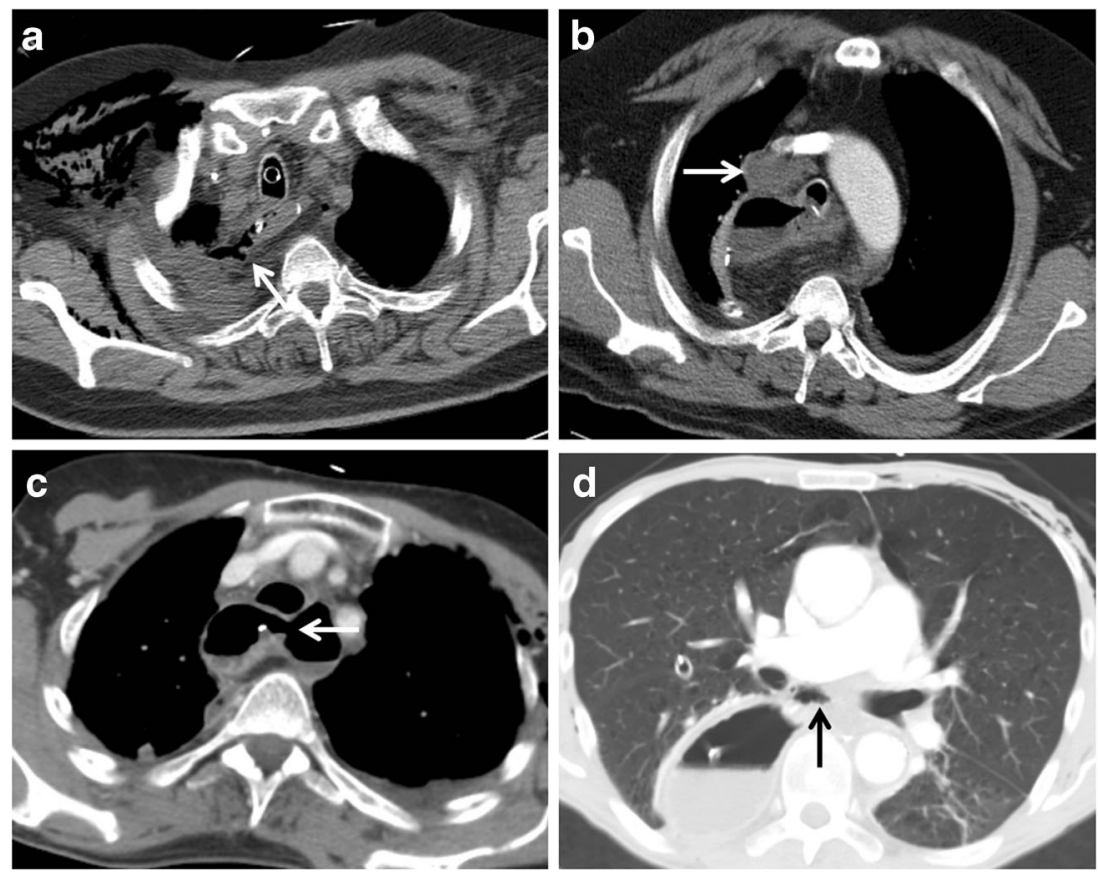
limitations and improve diagnostic accuracy of CT scanning after esophagectomy.

The developed ALP score has a good predictive value and includes well-recognized CT findings. The data indicate that, in the presence of two or more of four CT findings, the decision whether to start treatment could be made quite reliably, without true additional value of other diagnostic tests. This could lead to a reduction in treatment delay that is associated with additional tests. Although the cut-off point of $\geq 2$ yielded the highest overall discriminatory value, each additional point was associated with an increased risk, and clinical reasoning (particularly with scores of 1 and 3) remains important for treatment decision-making. To this regard, endoscopy after esophagectomy may be useful in cases where the results of the CT-scan are uncertain. Endoscopy has proven to be an accurate method to diagnose anastomotic leakage $[14,15]$.

In the current study in appeared counterintuitive that empyema fell out of the multivariable model, as it was highly predictive in univariable analysis. However, in multivariable prediction modeling the outcome (anastomotic leakage) is predicted based on values of a set of predictor variables (CT parameters). This method allows us to assess the impact of multiple predictor variables in the same model. In the current series at least two or more predictor variables that were highly suggestive for anastomotic leakage (i.e., fluid collection, air cavity, wall discontinuity, and fistula) were present in all 12 patients with empyema. Therefore, the added value of empyema - over the other predictor variables - for the prediction of anastomotic leakage was redundant in the current model, and therefore lost its significance. However, the fact remains that in clinical practice the presence of empyema on a postoperative CT scan is highly suggestive for the presence of an anastomotic leak.

Various limitations apply to this study. First, this study is limited by its retrospective nature. Second, the specific CT findings may be subject to interobserver variability. Standardization of mediastinal CT findings may overcome this problem. Finally, external validation of the prediction score is warranted since there is a risk of model overfitting due to multiple testing and differences with other patient populations (e.g., prevalence of leakage, use of other surgical techniques).

In conclusion, this study demonstrates that the presence of mediastinal fluid, mediastinal air, esophagogastric wall discontinuity and a fistula on a postoperative CT scan are independently and significantly associated with anastomotic leakage after esophagectomy in patients with a clinical suspicion of anastomotic leakage. Based on these items a CT-based anastomotic leakage prediction score was developed with superior discriminatory ability compared to systematic subjective $\mathrm{CT}$ assessment and original CT interpretation for the detection of anastomotic leakage after esophagectomy.

\section{Compliance with ethical standards}

Guarantor The scientific guarantor of this publication is Prof. R. van Hillegersberg.

Conflict of interest The authors of this manuscript declare no relationships with any companies, whose products or services may be related to the subject matter of the article.

Funding The authors state that this work has not received any funding.

Statistics and biometry Two of the authors have significant statistical expertise.

Ethical approval Institutional Review Board approval was obtained.

Informed consent Written informed consent was waived by the Institutional Review Board.

\author{
Methodology \\ - retrospective \\ - diagnostic \\ - observational \\ - performed at one institution
}

Open Access This article is distributed under the terms of the Creative Commons Attribution 4.0 International License (http:// creativecommons.org/licenses/by/4.0/), which permits unrestricted use, distribution, and reproduction in any medium, provided you give appropriate credit to the original author(s) and the source, provide a link to the Creative Commons license, and indicate if changes were made.

\section{References}

1. Torre LA, Bray F, Siegel RL, Ferlay J, Lortet-Tieulent J, Jemal A (2015) Global cancer statistics, 2012. CA Cancer J Clin 65:87-108

2. van Hagen P, Hulshof MC, van Lanschot JJ et al (2012) Preoperative chemoradiotherapy for esophageal or junctional cancer. N Engl J Med 366:2074-2084

3. Shapiro J, van Lanschot JJ, Hulshof MC et al (2015) Neoadjuvant chemoradiotherapy plus surgery versus surgery alone for oesophageal or junctional cancer (CROSS): long-term results of a randomised controlled trial. Lancet Oncol 16:1090-1098

4. Sjoquist KM, Burmeister BH, Smithers BM et al (2011) Survival after neoadjuvant chemotherapy or chemoradiotherapy for resectable oesophageal carcinoma: an updated meta-analysis. Lancet Oncol 12:681-692

5. Kim RH, Takabe K (2010) Methods of esophagogastric anastomoses following esophagectomy for cancer: a systematic review. J Surg Oncol 101:527-533

6. Saluja SS, Ray S, Pal S et al (2012) Randomized trial comparing side-to-side stapled and hand-sewn esophagogastric anastomosis in neck. J Gastrointest Surg 16:1287-1295

7. Urschel JD (1995) Esophagogastrostomy anastomotic leaks complicating esophagectomy: a review. Am J Surg 169:634-640

8. Iannettoni MD, Whyte RI, Orringer MB (1995) Catastrophic complications of the cervical esophagogastric anastomosis. J Thorac Cardiovasc Surg 110:1493-1500, discussion 1500-1

9. Kassis ES, Kosinski AS, Ross P Jr, Koppes KE, Donahue JM, Daniel VC (2013) Predictors of anastomotic leak after esophagectomy: an analysis of the society of thoracic surgeons general thoracic database. Ann Thorac Surg 96:1919-1926 
10. Boone J, Rinkes IB, van Leeuwen M, van Hillegersberg R (2008) Diagnostic value of routine aqueous contrast swallow examination after oesophagectomy for detecting leakage of the cervical oesophagogastric anastomosis. ANZ J Surg 78:784-790

11. Cools-Lartigue J, Andalib A, Abo-Alsaud A et al (2014) Routine contrast esophagram has minimal impact on the postoperative management of patients undergoing esophagectomy for esophageal cancer. Ann Surg Oncol 21:2573-2579

12. Tirnaksiz MB, Deschamps C, Allen MS, Johnson DC, Pairolero PC (2005) Effectiveness of screening aqueous contrast swallow in detecting clinically significant anastomotic leaks after esophagectomy. Eur Surg Res 37:123-128

13. Strauss C, Mal F, Perniceni T et al (2010) Computed tomography versus water-soluble contrast swallow in the detection of intrathoracic anastomotic leak complicating esophagogastrectomy (ivor lewis): a prospective study in 97 patients. Ann Surg 251:647-651

14. Maish MS, DeMeester SR, Choustoulakis E et al (2005) The safety and usefulness of endoscopy for evaluation of the graft and anastomosis early after esophagectomy and reconstruction. Surg Endosc 19:1093-1102

15. Page RD, Asmat A, McShane J, Russell GN, Pennefather SH (2013) Routine endoscopy to detect anastomotic leakage after esophagectomy. Ann Thorac Surg 95:292-298

16. Hogan BA, Winter DC, Broe D, Broe P, Lee MJ (2008) Prospective trial comparing contrast swallow, computed tomography and endoscopy to identify anastomotic leak following oesophagogastric surgery. Surg Endosc 22:767-771
17. Lantos JE, Levine MS, Rubesin SE, Lau CT, Torigian DA (2013) Comparison between esophagography and chest computed tomography for evaluation of leaks after esophagectomy and gastric pullthrough. J Thorac Imaging 28:121-128

18. Upponi S, Ganeshan A, D'Costa H et al (2008) Radiological detection of post-oesophagectomy anastomotic leak - a comparison between multidetector CT and fluoroscopy. Br J Radiol 81:545-548

19. Kauv P, Benadjaoud S, Curis E, Boulay-Coletta I, Loriau J, Zins M (2015) Anastomotic leakage after colorectal surgery: diagnostic accuracy of CT. Eur Radiol 25:3543-3551

20. Kim TH, Kim JH, Shin CI, Kim SH, Han JK, Choi BI (2015) CT findings suggesting anastomotic leak and predicting the recovery period following gastric surgery. Eur Radiol 25: 1958-1966

21. Bossuyt PM, Reitsma JB, Bruns DE et al (2015) STARD 2015: an updated list of essential items for reporting diagnostic accuracy studies. Radiology 277:826-832

22. Haverkamp L, van der Sluis PC, Verhage RJ, Siersema PD, Ruurda JP, van Hillegersberg R (2013) End-to-end cervical esophagogastric anastomoses are associated with a higher number of strictures compared with end-to-side anastomoses. J Gastrointest Surg 17:872-876

23. Steyerberg EW, Vickers AJ, Cook NR et al (2010) Assessing the performance of prediction models: a framework for traditional and novel measures. Epidemiology 21:128-138 